\title{
COPD-Patienten
}

\section{Wie hoch ist der jährliche FEV1-Abfall?}

\author{
Der jährliche FEV1-Abfall bei COPD- \\ Patienten 2. Grades liegt im Schnitt \\ bei $33 \mathrm{ml} / \mathrm{Jahr}$, bei Rauchern und bei \\ Patienten mit Lungenemphysem ist \\ er höher.
}

In der Publikation von Vestbo et al. wurden jetzt Teildaten der nicht-interventionellen ECLIPSE-Studie vorgestellt. Herausragendes Ergebnis war bei den COPD II'-Patienten ein durchschnittlicher FEV1Abfall von $33+2 \mathrm{ml} / \mathrm{Jahr}$ während des Beobachtungszeitraums von 3 Jahren. Allerdings variierten die Daten mit einer Abweichung von $59 \mathrm{ml} / \mathrm{Jahr}$ zwischen den Patienten erheblich. Bei Rauchern sank der FEV1-Abfall um 21+4 $\mathrm{ml}$ und bei NichtRauchern um 13+4 ml. Ein Lungenem-

physem war ebenfalls mit einem beschleunigten FEV1-Abfall assoziiert. Immerhin wiesen 23\% der Patienten ein $\triangle F E V 1$ von

J. Vestbo et al., Changes in forced expiratory volume in 1 second over time in COPD, NEJM, online publication 26. Sept. 2011

\section{"Bei Ex-Rauchern kleiner als gedacht.»}

Kommentar von Prof. A. Gillissen, Direktor der Klinik für Lungen- und Bronchialmedizin, Klinikum Kassel:Der FEV1-Abfall/ Jahr entspricht ungefähr dem, was man von großen interventionellen Studien wie UPLIFT oder TORCH kennt. Somit ist das hier publizierte Ergebnis keine Überraschung. Die einzige (reziproke) Assoziation von $\triangle \mathrm{FEV} 1$ ergibt sich mit dem Cla- minus bis plus $22 \mathrm{ml} / \mathrm{Jahr}$ auf. Bei $8 \%$ aller Patienten stieg die FEV1 um 20 ml/Jahr.

\section{Individuelle Unterschiede beim Asthma Kortison-Effekt ist genabhängig!}

\section{Durch die Identifizierung einer gene- tischen Variante, bei deren Vorliegen der Patient schlecht auf Steroide an- spricht, scheint ein weiterer Schritt in Richtung einer phänotypenspezifi- schen Therapie möglich.}

Viele Studien haben die genetische Basis eines Asthma bronchiale untersucht. In dieser Studie widmeten sich die Autoren dem Phänomen, dass Asthmapatienten unterschiedlich auf inhalative Kortikosteroide ansprechen und suchen nach dafür verantwortlichen Genmerkmalen, sog. SNPs (single-nucleotide polymorphisms).

Bei 935 Kindern (Alter 5-12 Jahre) wurde die pharmakogenetische Assoziation des SNP rs37972 untersucht. Es handelt sich um ein Allel, das für das Glukokortikoid-Transcript 1 Gen (GLCCI1) kodiert. Die Kinder wurden in folgende inhalative Behandlungsgruppen randomisiert: Budesonid/Triamcinolon/Flunisolid/Flucica-

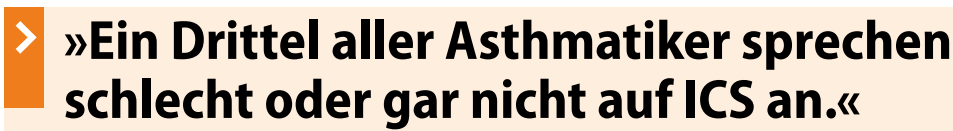

Kommentar von Prof. A. Gillissen, Direktor der Klinik für Lungen- und Bronchialmedizin, Klinikum Kassel: Es wäre von großem Vorteil, wenn man durch eine Vorabuntersuchung diejenigen Patienten selektieren könnte, die auf bestimmte Medikamente wie z.B. Kortikosteroide nicht oder nur schlecht ansprechen. Durch die Identifizierung einer genetischen Variante scheint ein weiterer Schritt in Richtung einer phänotypenspezfischen Therapie möglich. Die Studie imponiert durch ihre große Probandenzahl, die über 5 Jah-

son (ICS), Nedocromil, Placebo. Die FEV1Verbesserung wurde mit den genetischen Ergebnissen korreliert. Es zeigt sich, dass razellprotein (CC-16) ein Ergebnis, dass viele Hypothesen aufwirft. Letztendlich spiegelt die Studie Ergebnisse nur von Patienten mit einer COPD II $^{\circ}$ wider, dem vorgewählten Selektionskriterium. Wichtige Erkenntnis ist, dass es niemals zu spät ist, mit dem Rauchen aufzuhören, denn der FEV1-Abfall/Jahr der Ex-Raucher entsprach dem der Nicht-Raucher.

K.G.Tantisira et al. ; Genomwide association between GLCCI1 and response to glucocorticoid therapy in asthma, NEJM, online publication 26. Sept. 2011

re verfolgt und bei der eine Genom-weite SNP-Suche durchgeführt wurde. Zudem wurden auch noch Genproben von beiden Elternteilen gewonnen (DNA-Trios). Allerdings waren die FEV1-Unterschiede mit $3,2+1,6 \%$ (mit) vs. 9,4+9,41,1\% (ohne Variante) nicht überwältigend. Die Allelfrequenz der Variante kommt in der Allgemeinbevölkerung 0,4 - 16\% (Homozygotie für den Genotyp) vor und ist damit relativ selten. Der nächste Schritt müsste jetzt eine Studie sein, die in Praxis die Relevanz dieses Ergebnisses für die Therapieentscheidung belegt.

die GLCCI1 Variante mit einer 2,36-fach höheren Wahrscheinlichkeit eines Nichtansprechens auf ICS korrelierte. 